\title{
BMJ Open Retrospective cohort study of health service use for cardiovascular disease among adults with and without a record of injury hospital admission
}

\author{
Sean M Randall (D) , ${ }^{1}$ Fiona M Wood, ${ }^{2,3}$ Mark W Fear, ${ }^{2}$ James Boyd, ${ }^{4}$ \\ Suzanne Rea, ${ }^{2,3}$ Janine M Duke ${ }^{2}$
}

To cite: Randall SM, Wood FM Fear MW, et al. Retrospective cohort study of health service use for cardiovascular disease among adults with and without a record of injury hospital admission. BMJ Open 2020;10:e039104. doi:10.1136/ bmjopen-2020-039104

- Prepublication history and additional materials for this paper is available online. To view these files, please visit the journal online (http://dx.doi org/10.1136/bmjopen-2020039104).

Received 04 April 2020 Revised 22 September 2020 Accepted 29 September 2020

\section{Check for updates}

(c) Author(s) (or their employer(s)) 2020. Re-use permitted under CC BY-NC. No commercial re-use. See rights and permissions. Published by BMJ.

${ }^{1}$ School of Public Health, Curtin University, Perth, Western Australia, Australia

${ }^{2}$ Burn Injury Research Unit, Faculty of Health and Medical Sciences, The University of Western Australia, Perth, Western Australia, Australia ${ }^{3}$ Fiona Stanley Hospital and Perth Children's Hospital, Burns Service of Western Australia, Perth, Western Australia, Australia

${ }^{4}$ Department of Public Health, La Trobe University, Melbourne, Victoria, Australia

Correspondence to

Sean M Randall;

Sean.randall@curtin.edu.au

\section{ABSTRACT}

Objective To quantify postinjury cardiovascular-related health service use experienced by mid to older aged adults hospitalised for injury, compared with uninjured adults. Additionally, to explore the effect of beta-blocker medications on postinjury cardiovascular hospitalisations among injury patients, given the potential cardioprotective effects of beta blockers.

Design A retrospective cohort study using linked administrative and survey data.

Participants Records of 35026 injured and 60823 uninjured matched adults aged over 45 from New South Wales, Australia, who completed the 45 and up survey.

Primary and secondary outcome measures Admission rates and cumulative lengths of stay for cardiovascular hospitalisations, and prescription rates for cardiovascular medications. Negative binomial and Cox proportional hazards regression modelling were used to generate incident rate ratios (IRRs) and HR.

Results Compared with the uninjured, those with injury had a $19 \%$ higher adjusted rate of postinjury cardiovascular admissions (IRR 1.19, 95\% Cl 1.14 to 1.25), spent $40 \%$ longer in hospital for ardiovascular disease (IRR $1.40,95 \% \mathrm{Cl} 1.26$ to 1.57 ) and had slightly higher cardiovascular prescription rates (IRR 1.04, 95\% Cl 1.02 to 1.06), during study follow-up. Those in the injury cohort that used beta blockers both prior to and after injury (continuous) appeared to have reduced need for postinjury cardiovascular hospitalisation (IRR $1.09,95 \% \mathrm{Cl}$ 1.17 to 1.42) compared with those commencing on beta blockers after injury (after 30 days: IRR 1.69, 95\% Cl 1.37 to 2.08).

\section{Conclusions Apparent increased postinjury}

hospitalisation rates and prolonged length of stay related to cardiovascular disease suggest that injury patients may require clinical support for an extended period after injury. Additionally, injury patients who were on continuous beta blocker treatment appeared to have lower need for postinjury cardiovascular hospitalisations. However, the data do not allow us to draw clear conclusions and further clinical research is required.

\section{INTRODUCTION}

Trauma triggers systemic responses that can be divided into immunological, cardiovascular
Strengths and limitations of this study

- Availability of survey data for all participants allows use of additional information rarely found in administrative records, including detailed information on individual cardiovascular risk factors such as smoking, body mas index, physical activity levels, cholesterol, blood pressure and family history of cardiovascular disease.

- Availability of prescription information from the national prescription database allowed investigation of cardiovascular medication usage, including the ability to identify treatment for cardiovascular conditions in the primary care setting

- Observational design means it is difficult to provide any strong conclusions regarding the use of beta blockers after injury.

and metabolic. The pattern of these responses depends on whether the injury is mostly haemorrhagic, tissue damage or a combination of the two. ${ }^{1}$ Increased sympathetic activity is a critical part of the acute coordinated response to injury, modulating energy substrate mobilisation, cardiovascular and haemodynamic compensation and contributing to the host's immune response and healing. Cardiovascular responses are seen immediately after injury; immunological and inflammatory consequences are apparent several hours or days after the initial trauma, although these responses may also be triggered by very early cardiovascular changes. Metabolic responses are of greatest importance after successful resuscitation and treatment, although hypermetabolism triggered by severe burns is a significant issue. ${ }^{1}$

The response to injury in the acute phase has been well characterised and involves a rapid and coordinated elevation in the immune response enhanced by multiple pathways including elevated sympathetic 
nervous system activity, elevated catecholamine levels and a hypermetabolic response. More recently, evidence supports a moderate but sustained elevation of these pathways after recovery, with a proinflammatory milieu maintained for many years. ${ }^{2}{ }^{3}$ While short-term activation of stress response mechanisms is vital, the prolonged duration and increased magnitude of their activity leads to deleterious effects on metabolism, immune function and cardiovascular function. ${ }^{4}$ Cardiac stress mediated by increased plasma catecholamine levels (threefold or greater) after injury ${ }^{56}$ and activation of beta adrenoreceptors remains one of the main determinants of survival after injury. ${ }^{7}$ Additionally, elevated plasma catecholamines affect cellular immunity in the early phase of the trauma via the beta-adrenergic pathway. ${ }^{8-10}$

Beta-adrenoreceptor blockers (BBs), a class of medications commonly used to treat cardiovascular disease (CVD), have beneficial metabolic and cardioprotective effects $^{1112}$ and the use of BBs has been associated with improved outcomes in trauma patients. ${ }^{13} 14$ The interest in assessing beta blockade among injury patients is related to the hyperadrenergic state experienced by patients after injury. Recent evidence suggests that additional metabolic and immunomodulatory effects of BBs may provide benefit in managing injuries and their subsequent physiological effects, thereby expanding their clinical use in patients with trauma. ${ }^{5}$ While the precise mechanisms of improved injury patient outcomes associated with beta blockade reported are not clear, they may be the result of (1) reduction of periods of tachycardia which intensify myocardial oxygen demand and risk of ischaemia; (2) improved myocardial oxygen utilisation and (3) improved outcomes related to reversal of immunomodulatory properties mediated via the beta adrenoreceptor (figure 1).



Figure 1 Potential physiological links between injury and subsequent increased risk for CVD. Proposed mechanisms of beta-blockers and other cardiovascular therapy that may ameliorate the systemic responses in both the acute and chronic time frame and reduce CVD subsequent risk after injury. CVD, cardiovascular disease.
Our previous whole-of-population research has identified cardiovascular dysfunction after minor and severe burns $^{31516}$ and elevated rates of hospital admissions for CVD after burns and non-burn trauma compared with uninjured people for a prolonged period after the initial injury. ${ }^{17}$ The first aim of this study was to quantify postinjury CVD-related health service use (hospital admissions, length of hospital stay and prescription claims), controlling for sociodemographic, lifestyle, health and injury factors experienced by mid to older aged adults hospitalised for injury, compared with uninjured adults. The second aim was to explore whether the use of BBs in adult injury patients is related to reduced postinjury CVD hospitalisations, given the potential cardioprotective effects of these medications.

\section{METHODS}

\section{Study design, data source and study participants}

The study analysed self-reported and health administrative data, linked at an individual level, for a cohort of participants with a record of injury and a frequency matched cohort of uninjured participants identified in the 45 and Up Study. ${ }^{18}$

The Sax Institute's 45 and Up Study is a longitudinal cohort of 266885 participants aged 45 years and older in the state of NSW, Australia. Study participants were randomly sampled from the Australian Government Department of Human Services (DHS) enrolment database and recruited from 2006 to 2009. Details of the study methods are described elsewhere. ${ }^{18}$ In summary, study participants completed a baseline lifestyle and health survey (2006-2009) and consented to follow-up and linkage of their self-report data to routine health administrative databases. The overall response rate was $18 \% .^{18}$ The 45 and Up Study maintains an online repository of all published research. The study included around $11 \%$ of the NSW population aged 45 and over.

The data sources linked and included in this study: (1) the 45 and Up Study baseline survey (https://www. saxinstitute.org.au/our-work/45-up-study/); (2) the NSW Admitted Patient Care Data Collection (APDC), providing all hospital admissions in public and private hospital in NSW (2001-2018); (3) the Pharmaceutical Benefits Scheme (PBS) which provided information on subsidised prescription medicines (2005-2018) available to all Australian residents; (4) the Medical Benefits Schedule (MBS) which provided records of medical (general practitioner) services provided through Medicare, Australia's national health insurance scheme (2005-2018); and (5) the NSW Register of Births, Death and Marriages (2006-2018). Identification of 45 and Up Study participants' eligibility for this study (injury cohort; no injury cohort) and respective linkage of APDC and RBDM to survey data were conducted by the NSW Centre for Health Record Linkage (https://cherel.org.au). PBS and MBS data were linked by the Sax Institute using a unique identifier provided by DHS. 
The injury cohort comprised all individuals in the 45 and Up Study with a record of injury hospitalisation between their enrolment (occurring 2006-2009) and 2018, with no injury admission in previous 5 years (2001/2004-2006/2009). The uninjured comparison cohort comprised individuals randomly selected from the 45 and Up Study who did not have a record of an injury admission between 5 years before enrolment date (ie, 2001-2004) and 2018. Participants in the uninjured cohort were frequency matched $2: 1$ on age and gender to injury cases and were alive at the matching index date.

An injury admission was defined as a primary diagnosis of injury (International Statistical Classification of Diseases, 10th Revision, Australian Modification (ICD10-AM) S00T32) in the NSW APDC. The study index date is the date of injury admission.

\section{Patient and public involvement \\ No patient involved.}

\section{Study variables}

Injury

ICD10 codes were used to classify injury type, including fractures, open wounds, contusions and superficial injuries, dislocations, internal organ injuries, foreign bodies, amputations and burns (ICD codes found in online supplemental table 1). Injury severity was generated using survival risk ratios from NSW population-based injury hospital admissions data and applying published and widely used ICD-based Injury Severity Scaling (ICISS) methodology. ${ }^{19}$ The scores were classified as minor (ICISS $\geq 0.99$ ), moderate (ICISS $>0.941$ and $<0.99$ ) and severe (ICISS $\leq 0.941)$.

\section{Morbidity}

Hospital admissions principal diagnosis data were classified by disease group with ICD10-AM, for example, circulatory system (total-I00-I99) and by subgroups: ischaemic heart disease (IHD) (I20-25); heart failure (HF) (I50, I42.0, I42.6, I42.7, I42.9, I11.0), cerebrovascular disease (I60-I69). WHO Anatomical Therapeutic Chemical Classification (ATC) codes were used to classify PBS medications into anatomical and therapeutic groups ${ }^{20}$; Codes were classified at the first level (eg, anatomical groups 'A' Alimentary tract) with cardiovascular codes classified at the second level (eg, C07-BBs). Refer to online supplemental table 2) for a summary of ATC categories of cardiovascular medications. Time of supply of prescription was used to assess temporality of PBS medications, for example, prior: dispensed supply within a 4-month period prior to index date); post: dispensed supply after index date. Comorbidity was classified using the Charlson comorbidity index for 5 years of hospital data prior to the index admission. ${ }^{21}$ MBS data were used to count primary care (general pratictioner, GP) attendances and classify into quintiles. Indices of social disadvantage $^{22}$ and geographical remoteness ${ }^{23}$ were supplied for each study participant. Self-reported lifestyle variables (recorded at baseline) included smoking (current at time of survey, previous, never), alcohol (number of drinks per week prior to survey), body mas index (BMI) and physical activity (vigorous exercise, moderate exercise or neither in previous week).

\section{Outcomes measures}

Outcome measures include annual counts of CVD admissions and cumulative length of stay (LOS) and annual counts of prescriptions (total, CVD). The time until the first (incident) CVD admission and time until first CVD medication after study index were also used as outcome measures.

\section{Statistical analyses}

Descriptive statistics were generated for all study factors: sociodemographic (age, gender, social disadvantage, remoteness), pre-existing diseases, comorbidity, medication use, hospital admissions for CVD (prior, postinjury) and GP attendances, injury (type, severity) and self-reported lifestyle factors (smoking status; physical activity; BMI; alcohol consumption). Preliminary analyses included $\chi^{2}$ tests for categorical variables.

Outcome models to compare rates of postdischarge CVD health service use (prevalence) examined (1) annual total number of primary diagnosis CVD admissions (total and subgroups: IHD; HF and cerebrovascular disease) after study start; (2) annual cumulative LOS for CVD; and (3) prescription supply counts (total CVD 'C' ATC-code and subgroups). Incidence rate ratios (IRRs) and $95 \%$ CIs were calculated using multivariable negative binomial regression, adjusting for sociodemographic, injury (type, severity), comorbidity, medication use and other health and lifestyle factors.

To validate the above analysis, and in particular to control for potential differences in health status and health seeking behaviour, an additional analysis was performed using propensity score matching, comparing cardiovascular hospitalisation rates between the cohorts. Matching was chosen over alternative methods such as propensity score weighting as weighted methods can be vulnerable to outliers which can receive very high weights. ${ }^{24}$ The propensity analysis included all cases. Variables were selected for the propensity model based on their theoretical interest and strength of association with the outcome. The propensity score model was conducted to control for potential bias relating to previous health status and health seeking behaviour; as such, these variables were included first. Age was included as the strongest predictor of cardiovascular hospitalisation. Potential covariates were only included where propensity scores were balanced between blocks, and covariates were balanced across treatment and comparison groups within blocks, limiting the number of variables used; this was tested using the published 'PSCORE' algorithm. ${ }^{25}$ Age category, number of GP attendances in the year prior and existence of previous cardiovascular hospitalisations were included in the models. A logit model was used to 
calculate propensity scores. Matching was carried out by the simple (greedy) nearest neighbour method with one neighbour and no distance metric, with replacement. The average treatment effect on the treated (ATET) was used as the outcome.

Incidence of CVD: Adjusted HRs and 95\% CI were generated, adjusting for potential confounders to investigate the longitudinal relationship with time to first postinjury (1) CVD admission and (2) record of CVD medication. Analyses were conducted on cohort data that excluded those with a prior record of CVD (prescription, admission). In addition, analyses of first cardiovascular prescriptions were conducted on 0-30 days and $>30$ days after injury, to satisfy proportional hazards assumption tests. Mortality (all cause and CVD only) was examined using Cox proportional hazards regressions modelling.

\section{Assessment of BB use on postinjury CVD admissions among the injury cohort}

BB use in the injury cohort was classified as (1) continuous use (BB use during period 4 months prior to and 4 months after injury; new-onset BB use, with no BB use in 4-month period prior to injury and a; (2) record of $\mathrm{BB}$ use within 30 days of injury; (3) record of $\mathrm{BB}$ use within 60 days of injury; (4) record of BB use within 90 days of injury and (5) record of BB use within 120 days of injury. The time exposure of BB used in this study was based on clinical opinion, previous postinjury CVD research ${ }^{1617}$ and published papers examining BB use in acute phase after injury. ${ }^{432627}$ Multivariable negative binoimal regression was used to compare postinjury CVD admission rates for those with and without a record of $\mathrm{BB}$, adjusting for use of other cardivascular medications, sociodemographic, injury, health and lifestyle factors. Sensitivity analysis using linked cohort data from 2013 was undertaken to assess possible misclassification due to under copayment PBS agents prior to 2012. Multivariable negative binomial regresson using generalised estimating equation (GEE) modelling compared CVD hospitalisations in the 5-year preinjury and 5 year postinjury for each of the BB use categories. All analyses were carried using the Stata software package (v 16).

\section{RESULTS}

\section{Cohort characteristics}

This study identified 35026 individuals in the 45 and Up Study survey that had an injury hospitalisation between the date of baseline survey completion (2006-2008) and 2017. These individuals were frequency matched on age and gender to 60823 controls (uninjured) in the 45 and Up Study survey who did not have an injury hospitalisation from 5 years prior to survey completion to 2017. Injury cases for which no uninjured comparison individuals could be found $(n=272)$ were excluded from further analysis, resulting in 34754 cases.

The most common injury was fractures $(48 \%$, $\mathrm{n}=16832)$, followed by open wounds $(15 \%, \mathrm{n}=5100)$, contusions and superficial injuries $(8 \%, \mathrm{n}=2617)$, dislocations $(6 \%, \mathrm{n}=2101)$ internal organ injuries $(6 \%, \mathrm{n}=2088)$, foreign bodies $(2 \%, \mathrm{n}=599)$, amputations $(1 \%, \mathrm{n}=252)$ and burns $(1 \%, \mathrm{n}=222)$. Injury type was unspecified for $14 \%$ of cases $(n=4943)$. By injury severity, $38 \%(n=13269)$ were minor (ISS $>=0.99), 42 \% \quad(n=14517)$ were moderate $(0.941<=\mathrm{ISS}<0.99)$ and for $20 \% \quad(\mathrm{n}=6968)$ injury was severe (ISS <0.941). The median time spent in hospital with the index injury was 2 days (IQR $1-8$ days). The median length of follow-up for those with injury was 4.2 years (IQR 2.1-7.0 years) and for the uninjured, 4.2 years (IQR 2.1-6.9 years). A small number of the injury cohort $(2 \%, \mathrm{n}=691)$ died during the index admission. Of those with injury $27 \% \quad(n=9134)$ died before the end of the study date as compared with $19 \%$ of the uninjured study subjects $(\mathrm{n}=11571)$.

External causes of injury admissions: falls (63\%, $\mathrm{n}=21993)$, inanimate mechanical forces $(10 \%, \mathrm{n}=3591)$, transport $10 \% \quad(\mathrm{n}=3427)$ and 'animate' mechanical forces $3 \%(n=923)$ and burns $1 \%(n=222)$. Most of the remaining external causes were unspecified.

A comparison of the demographic and health characteristics of the two cohorts is shown in table 1. Notable differences included a greater proportion of those aged over 75 in the injury group, caused by an inability to find multiple controls for each case in this age group during the extraction phase. The health status of those with an injury hospitalisation was generally worse, with higher Charlson comorbidity, rates of previous CVD hospitalisations and greater number of medications used.

\section{Post injury CVD health service use (admissions, LOS and prescription use)}

Of those with an injury, 22\% ( $\mathrm{n}=7791)$ had a hospitalisation within the follow-up period where CVD was the primary diagnosis, for a total of 15445 CVD hospitalisations. Most individuals $(\mathrm{n}=4601,59 \%)$ with a CVD hospitalisation had only one, $37 \%(\mathrm{n}=2858)$ between 2 and 5 CVD hospitalisations and 4\% ( $\mathrm{n}=332)$ had more than five CVD admissions. In the uninjured comparison group, $16 \%(\mathrm{n}=9547)$ had at least one CVD hospitalisation after the matched index date; there were 16781 CVD hospitalisations in total in this group, with a similar distribution. Admissions for IHD were the most common CVD diagnosis in both injury cases and uninjured controls; refer to table 2 for details of cardiovascular subconditions.

The unadjusted (observed) annual incidence rates of CVD admissions (total, specific subconditions) for the two cohorts are shown in figure 2. These graphs show higher admission rates for the injury cohort across all cardiovascular conditions for the entire follow-up period of the study. The difference was also observable prior to the index injury, indicating differences in the underlying health of these cohorts.

After adjustment for a range of sociodemographic and pre-existing health factors, the injury cohort had a 19\% higher rate of postinjury CVD admissions over the follow-up period, compared with those without injury 
Table 1 Baseline demographic and pre-existing health status factors for those with a first burn injury hospitalisation, and non-injured cohort, Western Australia, 2000-2012

\begin{tabular}{|c|c|c|c|}
\hline Characteristics & $\begin{array}{l}\text { No injury } N \\
(\%)\end{array}$ & Injury N (\%) & $P$ value \\
\hline Total & 60823 & 34754 & \\
\hline \multicolumn{4}{|l|}{ Demographic } \\
\hline \multicolumn{4}{|l|}{ Sex } \\
\hline Male & 31655 (52.0) & $18380(52.9)$ & 0.012 \\
\hline \multicolumn{4}{|l|}{ Age } \\
\hline$<60$ & $12970(21.3)$ & $6458(18.6)$ & $<0.001$ \\
\hline $60-74$ & 23397 (38.5) & $11636(33.5)$ & \\
\hline $75+$ & $24456(40.2)$ & $16660(47.9)$ & \\
\hline \multicolumn{4}{|c|}{ Social disadvantage quintiles* } \\
\hline $\begin{array}{l}\text { Quintile 1. (Most } \\
\text { disadvantaged) }\end{array}$ & $12983(21.4)$ & $7669(22.1)$ & $<0.001$ \\
\hline Quintile 2. & $13183(21.7)$ & $7244(20.8)$ & \\
\hline Quintile 3. & $11086(18.2)$ & $6242(18.0)$ & \\
\hline Quintile 4. & 10075 (16.6) & $5609(16.1)$ & \\
\hline $\begin{array}{l}\text { Quintile 5. (least } \\
\text { disadvantaged) }\end{array}$ & $11870(19.5)$ & $7129(20.5)$ & \\
\hline \multicolumn{4}{|l|}{ Remoteness $†$} \\
\hline Major city & $31381(51.6)$ & $19476(56.0)$ & $<0.001$ \\
\hline Inner regional & $21579(35,5)$ & 11098 (31.9) & \\
\hline Outer regional & $6231(10.2)$ & $3293(9.5)$ & \\
\hline Remote & $465(0.8)$ & $245(0.7)$ & \\
\hline Very remote & $67(0.1)$ & $38(0.1)$ & \\
\hline \multicolumn{4}{|l|}{ Health status } \\
\hline $\begin{array}{l}\text { Any comorbidity (CCl } \\
\geq 1) \ddagger\end{array}$ & $18695(30.7)$ & $14603(42.0)$ & $<0.001$ \\
\hline $\begin{array}{l}\text { Any circulatory system } \\
\text { hospitalisation in } \\
\text { previous } 5 \text { years }\end{array}$ & $15893(26.1)$ & $13203(38.0)$ & $<0.001$ \\
\hline $\begin{array}{l}\text { Any IHD hospitalisation } \\
\text { in previous } 5 \text { years }\end{array}$ & $4735(7.8)$ & $3971(11.4)$ & $<0.001$ \\
\hline $\begin{array}{l}\text { Any cerebrovascular } \\
\text { hospitalisation in } \\
\text { previous } 5 \text { years }\end{array}$ & $1840(3.0)$ & $2006(5.8)$ & $<0.001$ \\
\hline $\begin{array}{l}\text { Any heart failure } \\
\text { hospitalisation in } \\
\text { previous } 5 \text { years }\end{array}$ & 2022 (3.3) & $2174(6.3)$ & $<0.001$ \\
\hline \multicolumn{4}{|l|}{ Self-reported information } \\
\hline Have diabetes & $5796(9.5)$ & $4051(11.7)$ & $<0.001$ \\
\hline Had blood clot & $2826(4.7)$ & $2333(6.7)$ & 0.003 \\
\hline High blood pressure & 23605 (38.8) & $13951(40.1)$ & $<0.001$ \\
\hline Heart disease & $8341(13.7)$ & $5853(16.8)$ & $<0.001$ \\
\hline \multicolumn{4}{|l|}{ BMI, $\mathrm{kg} / \mathrm{m}^{2 \star}$} \\
\hline Underweight $(<18.5)$ & $4934(8.1)$ & $3299(9.5)$ & $<0.001$ \\
\hline Normal (18.5-25) & 20915 (34.4) & $12234(35.2)$ & \\
\hline Overweight (25-30) & 22727 (37.4) & $12195(35.1)$ & \\
\hline Obese class 1 (30-35) & $8582(14.1)$ & $4704(13.5)$ & \\
\hline Obese class $2(35-40)$ & $2272(3.7)$ & $1429(4.1)$ & \\
\hline
\end{tabular}

Continued
Table 1 Continued

\begin{tabular}{crrr} 
Characteristics & $\begin{array}{l}\text { No injury N } \\
(\%)\end{array}$ & Injury N (\%) & P value \\
\hline $\begin{array}{l}\text { Obese class 3+ } \\
(40-50)\end{array}$ & $844(1.4)$ & $538(1.6)$ & \\
$\begin{array}{l}\text { Smoking and alcohol } \\
\text { Current smoker }\end{array}$ & $3660(6.0)$ & $2309(6.6)$ & $<0.001$ \\
\hline Previous smoker & $21829(35.9)$ & $12710(36.6)$ & \\
\hline Never smoker & $35334(58.1)$ & $19735(56.8)$ & \\
\hline 8+ drinks per week & $18320(30.2)$ & $10278(29.7)$ & 0.088 \\
\hline
\end{tabular}

8+ drinks per week $18320(30.2) \quad 10278(29.7) \quad 0.088$

Primary care (no of GP visits in previous year)

$\begin{array}{lrrr}0-3 & 15115(24.9) & 6559(18.9) & <0.001 \\ 4-6 & 13224(21.7) & 5990(17.2) & \\ 7-10 & 12936(21.3) & 6790(19.5) \\ 11-16 & 10776(17.7) & 6972(20.1) \\ 17+ & 8772(14.4) & 8443(24.3) & \end{array}$

Physical activity

Vigorous exercise in $16574(29.8) \quad 8984(28.1) \quad<0.001$ week

Moderate only exercise 27482 (49.5) 15213 (47.6) in week

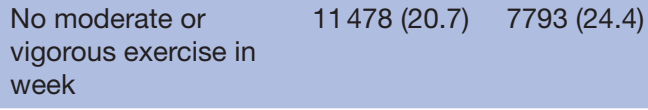

Family history

$\begin{array}{llll}\text { Heart disease } & 27847(45.8) & 16110(46.4) & 0.089 \\ \text { High blood pressure } & 29373(48.3) & 15870(45.7) & <0.001 \\ \text { Stroke } & 16248(26.7) & 9351(26.9) & 0.518\end{array}$

PBS medication use in 4 months prior to injury (ATC code)

\begin{tabular}{|c|c|c|c|}
\hline $\begin{array}{l}\text { Alimentary tract/ } \\
\text { metabolism }(\mathrm{A})\end{array}$ & $21972(36.1)$ & $15013(43.2)$ & $<0.001$ \\
\hline Blood/blood forming & $5637(9.3)$ & $5437(15.6)$ & $<0.001$ \\
\hline $\begin{array}{l}\text { Platelet aggregation } \\
\text { inhibitors (B) }\end{array}$ & $5473(9.0)$ & $4323(12.4)$ & $<0.001$ \\
\hline $\begin{array}{l}\text { Cardiovascular } \\
\text { medications (C) }\end{array}$ & $34973(57.5)$ & $21142(60.8)$ & $<0.001$ \\
\hline Cardiac therapy & $4556(7.5)$ & $3770(10.9)$ & $<0.001$ \\
\hline Antihypertensives & $1805(3.0)$ & $1155(3.3)$ & 0.002 \\
\hline Diuretics & $5178(8.5)$ & 4459 (12.8) & $<0.001$ \\
\hline Peripheral vasodilators & 0 & 0 & - \\
\hline Vasoprotectives & 0 & 0 & - \\
\hline Beta blockers & $8193(13.5)$ & $5514(15.9)$ & $<0.001$ \\
\hline $\begin{array}{l}\text { Calcium channel } \\
\text { blockers }\end{array}$ & 7832 (12.9) & $5081(14.6)$ & $<0.001$ \\
\hline $\begin{array}{l}\text { Renin/angiotensin } \\
\text { agents }\end{array}$ & $22917(37.7)$ & $13308(38.3)$ & 0.06 \\
\hline Lipid-modifying agents & $22136(36.4)$ & $13244(38.1)$ & $<0.001$ \\
\hline Dermatologicals (D) & $4610(7.6)$ & $3204(9.2)$ & $<0.001$ \\
\hline Genitourinary (G) & $3762(6.2)$ & $2344(6.7)$ & 0.001 \\
\hline Hormonal $(\mathrm{H})$ & $5553(9.1)$ & $4262(12.3)$ & $<0.001$ \\
\hline Anti-infectives (J) & $14621(24.0)$ & 11094 (31.9) & $<0.001$ \\
\hline $\begin{array}{l}\text { Antineoplastic/immune } \\
\text { modulating drugs (L) }\end{array}$ & $2363(3.9)$ & $1600(4.6)$ & $<0.001$ \\
\hline
\end{tabular}




\begin{tabular}{lccc}
\hline Table 1 Continued & & \\
\hline Characteristics & $\begin{array}{l}\text { No injury N } \\
\text { (\%) }\end{array}$ & Injury N (\%) & P value \\
\hline Musculoskeletal (M) & $11293(18.6)$ & $8287(23.8)$ & $<0.001$ \\
\hline Nervous system (N) & $18901(31.1)$ & $16205(46.6)$ & $<0.001$ \\
Antiparasitics (P) & $86(0.1)$ & $77(0.2)$ & 0.004 \\
Respiratory (R) & $7095(11.7)$ & $5056(14.6)$ & $<0.001$ \\
Sensory organs (S) & $9262(15.2)$ & $6512(18.7)$ & $<0.001$ \\
Various (V) & $396(0.6)$ & $323(0.9)$ & $<0.001$ \\
\hline
\end{tabular}

*Socio-Economic Indexes for Areas (SEIFA) socioeconomic disadvantage quintiles; missing values $2.5 \%$ injury, $2.7 \%$ no injury. †Accessibility/Remoteness Index of Australia (ARIA+) remoteness classification; missing values $1.7 \%$ injury, $1.8 \%$ no injury. $\ddagger$ Based on derived Charlson Comorbidity Index (CCl) using 5-year look-back hospitalisations.

ATC, Anatomical Therapeutic Chemical Classification; BMI, body mas index; GP, general pratictioner; IHD, ischaemic heart disease; PBS, Pharmaceutical Benefits Scheme.

Table 2 Post injury (study index) count of CVD hospitalisations and medication use for adults in injury versus uninjured cohorts

\begin{tabular}{|c|c|c|}
\hline & $\begin{array}{l}\text { No injury } \\
\text { cohort }\end{array}$ & $\begin{array}{l}\text { Injury } \\
\text { cohort }\end{array}$ \\
\hline $\begin{array}{l}\text { CVD hospital admissions disease } \\
\text { subgroup* }\end{array}$ & \multicolumn{2}{|c|}{ Hospitalisation count } \\
\hline Rheumatic fever & 73 & 58 \\
\hline Essential hypertension & 279 & 327 \\
\hline Ischaemic heart disease & 4750 & 3827 \\
\hline Pulmonary heart disease & 469 & 407 \\
\hline Other heart disease & 4128 & 3330 \\
\hline Heart failure & 2415 & 2844 \\
\hline Cerebrovascular disease & 1944 & 1978 \\
\hline Disease of arteries & 1132 & 1050 \\
\hline Disease of veins & 1088 & 902 \\
\hline Other circulatory diseases & 503 & 722 \\
\hline $\begin{array}{l}\text { CVD medications by ATC } \\
\text { subgroups } \dagger\end{array}$ & \multicolumn{2}{|c|}{ Prescription count } \\
\hline Cardiac therapy (C01) & 125790 & 122109 \\
\hline Antihypertensives (C02) & 56811 & 32513 \\
\hline Diuretics (C03) & 85338 & 87686 \\
\hline Beta blocking agents (C07) & 236605 & 159882 \\
\hline Calcium channel blockers (C08) & 300028 & 199330 \\
\hline $\begin{array}{l}\text { Agents-renin-angiotensin system } \\
\text { (C09) }\end{array}$ & 1038724 & 594538 \\
\hline Lipid-modifying agents (C10) & 938813 & 557714 \\
\hline
\end{tabular}

${ }^{*}$ CVD primary diagnosis uisng ICD codes in hospital admissions data.

†ldentified in PBS claim data.

ATC, Anatomical Therapeutic Chemical Classification; CVD, cardiovascular disease; ICD, International Statistical Classification of Diseases; PBS, Pharmaceutical Benefits Scheme.
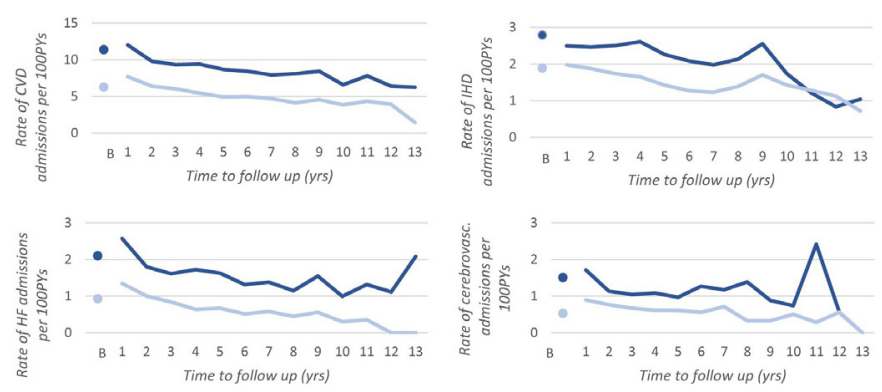

Figure 2 Unadjusted rates of cardiovascular hospitalisations and cardiovascular subconditions after index date for those with and without an index injury. $B$ (x-axis) represents a baseline rate 1 year prior to injury. CVD, cardiovascular disease; HF, heart failure; IHD, ischaemic heart disease.

(IRR 1.19, 95\% CI 1.14 to 1.25). Results from the adjusted negative binomial regression are shown in table 3. Age was the most important predictor of increased CVD admissions, followed by a history of CVD admissions in the previous 5 years; males had 1.45 times greater risk than females. Use of lipid-modifying agents, living in inner and outer regional areas, and self-reported higher levels physical activity and family history of high blood pressure were protective against postinjury CVD admissions. With respect to LOS, the injury cohort spent $40 \%$ longer in hospital with a CVD as compared with those without injury (IRR $1.40,95 \%$ CI 1.26 to 1.57). Examination of subcohorts of injury type showed significantly elevated LOS for those with fractures (IRR 1.17, 95\% CI 1.09 to 1.24) and open wound injuries (IRR $1.18,95 \%$ CI 1.07 to 1.31 ), the two most common types of injuries in the study, while a larger effect was seen in those with burn injuries (IRR $1.48,95 \%$ CI 1.02 to 2.15 ). When examining each injury severity group, the results were similar: minor (IRR 1.20, 95\% CI 1.11 to 1.28 ); moderate (IRR $1.16,95 \%$ CI 1.09 to 1.24); and, severe (IRR $1.20,95 \%$ CI 1.11 to 131 ). Propensity score matching was carried out comparing 34754 with 34754 matched controls. There were no missing values on any of variables of interest. The covariates of interest were balanced after matching, with standardised differences of less than 0.01 between cohorts. The ATET was 0.13 (95\% CI 0.11 to 0.15 ) additional cardiovascular hospitalisations for those with an injury.

The injury cohort had higher admission rates for IHD (IRR $1.30,95 \%$ CI 1.16 to 1.45 ), HF (IRR $1.14,95 \%$ CI 1.05 to 1.23 ) and cerebrovascular disease (IRR 1.22, 95\% CI 1.07 to 1.40), when compared with the uninjured cohort. For the burn subcohort, the CVD subgroup results did not reach significance, reflecting the small number in this study with burns (IRR, 95\% CI: IHD:1.40, 0.66 to 2.96; HF: $1.37,0.75$ to 2.47 ; cerebrovascular disease: 0.39 , 0.10 to 1.42 ).

In the injury cohort, $72 \%(\mathrm{n}=25044)$ had a record of a CVD prescription within the follow-up period (1.75 million prescription records in total) while $70 \%$ $(n=42604)$ of the uninjured cohort had a record of a CVD 
Table 3 Postinjury CVD hospitalisations: adjusted incidence rate ratios (IRRs) and 95\% Cls injured versus uninjured adults

\begin{tabular}{|c|c|c|c|}
\hline Characteristics & IRR & Lower $\mathrm{Cl}$ & Upper $\mathrm{Cl}$ \\
\hline Injury (yes vs no) & 1.19 & 1.14 & 1.26 \\
\hline \multicolumn{4}{|l|}{ Gender } \\
\hline Male & 1.00 & - & - \\
\hline Female & 0.69 & 0.65 & 0.73 \\
\hline \multicolumn{4}{|l|}{ Age } \\
\hline $45-49$ & 1.00 & - & - \\
\hline $50-54$ & 1.13 & 0.85 & 1.51 \\
\hline $55-59$ & 1.32 & 1.00 & 1.75 \\
\hline $60-64$ & 1.86 & 1.42 & 2.45 \\
\hline $65-69$ & 2.30 & 1.75 & 3.04 \\
\hline $70-74$ & 2.91 & 2.22 & 3.82 \\
\hline $75-79$ & 3.64 & 2.77 & 4.79 \\
\hline $80-84$ & 4.63 & 3.54 & 6.08 \\
\hline $85+$ & 5.39 & 4.10 & 7.07 \\
\hline \multicolumn{4}{|l|}{ Remoteness level } \\
\hline Major city & 1.00 & - & - \\
\hline Inner regional & 0.93 & 0.89 & 0.98 \\
\hline Outer regional & 0.88 & 0.82 & 0.95 \\
\hline Remote & 1.19 & 0.97 & 1.46 \\
\hline Very remote & 0.80 & 0.44 & 1.42 \\
\hline \multicolumn{4}{|l|}{ Health status } \\
\hline $\begin{array}{l}\text { Hospitalisation for } \\
\text { cardiovascular disease } \\
\text { in previous } 5 \text { years }\end{array}$ & 1.66 & 1.55 & 1.78 \\
\hline $\begin{array}{l}\text { Charlson Comorbidity } \\
\text { Index } \geq 1\end{array}$ & 1.37 & 1.28 & 1.46 \\
\hline
\end{tabular}

CVD medication prior 4 months to study start (ATC code)

\begin{tabular}{llll} 
Blood thinners (B01) & 1.19 & 1.11 & 1.28 \\
Cardiac therapy (C01) & 1.41 & 1.31 & 1.51 \\
Antihypertensives (C02) & 1.28 & 1.11 & 1.49 \\
Diuretics (C03) & 1.45 & 1.35 & 1.56 \\
Beta blockers (C07) & 1.23 & 1.15 & 1.31 \\
$\begin{array}{l}\text { Calcium channel } \\
\text { blockers (C08) }\end{array}$ & 1.10 & 1.03 & 1.17 \\
$\begin{array}{l}\text { Renin-angiotensin (C09) } \\
\text { Lipid-modifying agents }\end{array}$ & 0.89 & 0.97 & 1.08 \\
(C10) & & & 0.84 \\
\hline
\end{tabular}

No GP visits in previous year

\begin{tabular}{llll}
\hline $0-3$ & 1.00 & & \\
\hline $4-6$ & 1.05 & 0.97 & 1.13 \\
\hline $7-10$ & 1.14 & 1.06 & 1.23 \\
\hline $11-16$ & 1.22 & 1.13 & 1.32 \\
\hline $17+$ & 1.38 & 1.26 & 1.51 \\
Self-report information & & & \\
\hline \begin{tabular}{l} 
Physical activity \\
\hline
\end{tabular} & & & \\
\hline
\end{tabular}

Continued

\begin{tabular}{|c|c|c|c|}
\hline Characteristics & IRR & Lower $\mathrm{Cl}$ & Upper Cl \\
\hline Minimal & 1.00 & - & - \\
\hline Moderate & 0.92 & 0.87 & 0.97 \\
\hline Vigorous & 0.91 & 0.85 & 0.97 \\
\hline \multicolumn{4}{|l|}{$\mathrm{BMI}, \mathrm{kg} / \mathrm{m}^{2}$} \\
\hline$<18.5$ & 1.00 & - & - \\
\hline $18.5-25$ & 0.90 & 0.83 & 0.97 \\
\hline $25-30$ & 0.95 & 0.88 & 1.03 \\
\hline 30-35 & 1.05 & 0.96 & 1.15 \\
\hline $35-40$ & 1.09 & 0.97 & 1.24 \\
\hline $40+$ & 1.29 & 1.05 & 1.57 \\
\hline \multicolumn{4}{|l|}{ Smoking } \\
\hline Never smoked & 1.00 & - & - \\
\hline Previously smoked & 1.10 & 1.05 & 1.16 \\
\hline Currently smoke & 1.35 & 1.24 & 1.48 \\
\hline \multicolumn{4}{|c|}{ Diagnosis - told by doctor } \\
\hline Diabetes & 1.12 & 1.04 & 1.19 \\
\hline High blood pressure & 1.17 & 1.11 & 1.23 \\
\hline Blood clot & 1.13 & 1.05 & 1.23 \\
\hline Heart condition & 1.53 & 1.45 & 1.62 \\
\hline \multicolumn{4}{|l|}{ Family history } \\
\hline High blood pressure & 0.92 & 0.87 & 0.96 \\
\hline Stroke & 1.05 & 0.99 & 1.11 \\
\hline Heart disease & 1.17 & 1.11 & 1.22 \\
\hline
\end{tabular}

BMI, body mas index; CVD, cardiovascular disease; GP, general pratictioner.

prescription (2.78 million prescription records in total). A summary of cardiovascular prescriptions by subclass is shown in table 2; medications acting on the renin-angiotensin system and lipid-modifying agents were the most common subclasses of medications used by both injured and uninjured cohorts.

After adjustment for health, injury, lifestyle and sociodemographic factors, there was a significant but minimal increase in cardiovascular prescriptions (total) rates in those with injury when compared with uninjured adults (IRR 1.04, 95\% CI 1.02 to 1.06). However, this result concealed larger differences found for specific medication subclasses. Those with injury had significantly higher postinjury prescription rates when compared with the uninjured cohort for cardiac therapy medications (IRR $1.27,95 \%$ CI 1.18 to 1.38 ), diuretics (IRR $1.40,95 \%$ CI 1.32 to 1.48 ), BBs (IRR $1.21,95 \%$ CI 1.14 to 1.28 ), calcium channel blockers (IRR 1.25, 95\% CI 1.17 to 1.33), than for agents acting on the renin-angiotensin system (IRR 1.05, 95\% CI 1.02 to 1.08 ) and lipid-modifying agents (IRR $1.04,95 \%$ CI 1.01 to 1.08$)$. There was no difference found for antihypertensives (IRR 1.00, 95\% CI 0.87 to 1.16). 
New onset (incident) cardiovascular hospitalisations and prescriptions

Incident cardiovascular hospitalisations

Individuals in both cohorts who had CVD hospitalisations in the previous 5 years, cardiovascular medication prescriptions in the previous 5 years, or had self-reported a cardiovascular condition in the 45 and Up survey results were excluded. There were 7274 subjects with an injury in this reduced cohort, and 15608 uninjured control subjects. A total of 781 (10.7\%) individuals in the reduced injury cohort had a first-time CVD hospitalisation in the study period, while 2208 (30.4\%) had a first-time cardiovascular prescription. In the reduced uninjured cohort, the number with a first-time CVD hospitalisation was 1119 $(7.2 \%)$, with $4161(26.7 \%)$ having a record of a first-time cardiovascular prescription.

After adjustment for confounders, the injury cohort had a $36 \%$ greater rate of first time CVD admissions (HR $1.36,95 \%$ CI 1.23 to 1.50 ) compared with the uninjured cohort. The injury cohort also had significantly higher rates for first admissions for IHD (HR 1.24, 95\% CI 1.04 to 1.48 ) and cerebrovascular disease (HR 1.43, 9\% CI 1.11 to 1.85 ); however, HF (HR 1.03, 95\% CI 0.67 to 1.57 ) was not significantly greater.

\section{Incident cardiovascular prescriptions}

During the first 30-day period after injury, compared with the uninjured, the injury cohort had a 2.6 (HR $2.59,95 \%$ CI 1.94 to 3.47 ) times higher adjusted rate of first time cardiovascular (combined) prescriptions, with significantly higher prescription rates for each cardiovascular medication subgroup except antihypertensives, when compared with the uninjured. After 30 days, there was a small but significant increase in first cardiovascular prescriptions in those with an injury (HR 1.07, 95\% CI 1.01 to 1.13 ) compared with uninjured. Subgroups of cardiovascular medications with higher rates of first prescriptions included those for cardiac therapy (includes cardiac glycosides, antiarrhythmic, adrenergic vasodilators (organic nitrates) agents), diuretics and BBs. Refer to table 4 for summary of results.

\section{BB medication use and CVD hospitalisations in those with injury}

There were 5514 individuals in the injury cohort who had records of $\mathrm{BB}$ prescriptions in the 4 months before injury; of these, 4329 had a BB prescription filled in the 4 months after injury, suggesting continuous use. There were 432 individuals in the injured cohort who commenced BBs in the thirty days after injury, with no use in the prior 4 months (this increased to 619 individuals commencing BBs in the 60 days after injury, 797 commencing BBs in 90 days after injury, and 928 commencing BB in the first 120 days after injury). There was no record of BB usage in the 4 months prior/post injury in $81 \%$ of the injury cohort $(n=28312)$. Unadjusted rates of hospitalisations for those in the injury cohort by cardiovascular medication subgroups (continuous BB, first BB in 30 days
Table 4 Postinjury first time (incident) cardiovascular prescriptions for injured versus uninjured adults: adjusted $\mathrm{HR}$ and $95 \% \mathrm{Cl}$

\begin{tabular}{|c|c|c|}
\hline Medication group & $\begin{array}{l}\text { HR, } 95 \% \mathrm{Cl} 0 \text { to } 30 \\
\text { days after injury } \\
\text { (study index) }\end{array}$ & $\begin{array}{l}\mathrm{HR}, 95 \% \mathrm{CI}>30 \\
\text { days after injury } \\
\text { (study index) }\end{array}$ \\
\hline $\begin{array}{l}\text { Cardiovascular } \\
\text { medications } \\
\text { (combined) }\end{array}$ & $2.59,1.94$ to 3.47 & $1.07,1.01$ to 1.13 \\
\hline Cardiac therapy & $5.51,1.73$ to 17.52 & $1.17,1.01$ to 1.35 \\
\hline Antihypertensives & $0.56,0.10$ to 3.19 & $1.13,0.88$ to 1.46 \\
\hline Diuretics & $5.50,2.74$ to 11.04 & $1.47,1.29$ to 1.68 \\
\hline Beta blockers & $3.99,1.77$ to 8.99 & $1.16,1.03$ to 1.32 \\
\hline $\begin{array}{l}\text { Calcium channel } \\
\text { blockers }\end{array}$ & $10.43,3.00$ to 36.29 & $1.10,0.94$ to 1.29 \\
\hline $\begin{array}{l}\text { Renin-angiotensin } \\
\text { agents }\end{array}$ & $2.40,1.46$ to 3.94 & $0.00,0.92$ to 1.08 \\
\hline Lipid modifiers & $1.79,1.04$ to 3.07 & $1.08,0.99$ to 1.17 \\
\hline
\end{tabular}

after injury, no BB) are shown in figure 3 . For the 2 years prior to injury (study index), those in the continuous BB group had higher CVD admission rates than those commencing BB after injury. However, after injury the observed annual rates of CVD admission for continuous BB users declined and were lower than that observed for those who commenced BB after injury. For those who commenced $\mathrm{BB}$ after injury, the preinjury steep incline of CVD admissions continued to a peak at 1-year after injury, after which time the CVD admission rates declined but remained higher than that observed for continuous BB users for a further 3.5 years. For a summary of IRR (95\% CI) results (adjusting for sociodemographic, health, injury and medication use factors), comparing those in the injury cohort with record of continuous BB (4 months preinjury and 4 months postinjury), BB commenced after index injury (within 30 days, 60 days, 90 days, 120 days)) vs those with no record of BB for (1) all cohort data and (2) data from 2013 onwards, refer to table 5 . The results in table 5 did not identify any difference in results using the total cohort (2006+) or reduced cohort (2013+), that is, no misclassification bias due to under-copayment medications was identified.

In addition, adjusted negative binomial regression using GEE modelling that examined CVD hospitalisations in 5 years after injury with 5 years preinjury for each category of BB use versus no BB use, found similar effects of lower post-injury CVD hospitalisations for continuous $\mathrm{BB}$ compared with those commencing $\mathrm{BB}$ after injury: continuous BB use (IRR 1.11, 95\% CI 1.06 to 1.16 ); BB started within 30 days (IRR 1.32, 95\% CI 1.19 to 1.47 ), 60 days (IRR 1.31, 95\% CI 1.19 to 1.43 ), 90 days (IRR $1.28,95 \%$ CI 1.18 to 1.40 ), 120 days (IRR $1.21,95 \%$ CI 1.12 to 1.31). Refer to figure 4 for number of CVD hospitalisations per person for the 5-year windows before and after injury; only those on continuous BB (before and after injury) experienced a decrease in post injury CVD admissions. 


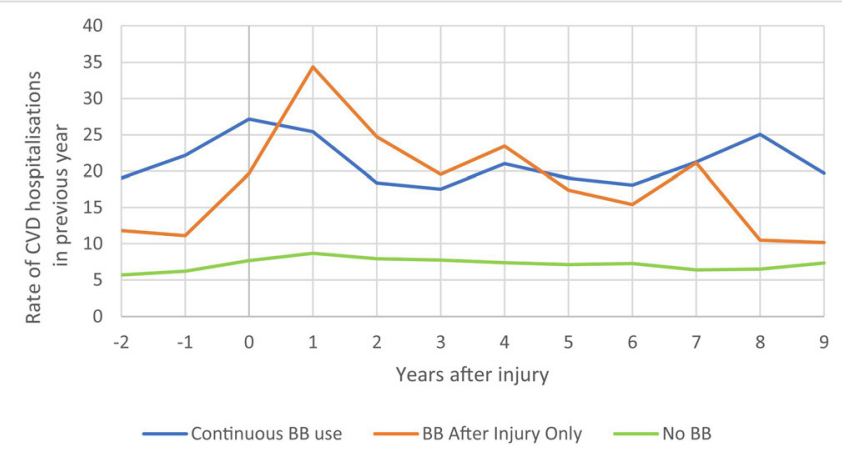

Figure 3 Unadjusted rates of cardiovascular hospitalisations in those with injury by cardiovascular medication groups; (A) continuous BB use-those with BB use in the 4 months before/after injury; (B) those with no prior BB use but BB prescription in 30 days after injury and $(C)$ those with no record of $\mathrm{BB}$ prescription in 4 months before/after injury. BB, Beta blocker; CVD, cardiovascular disease.

\section{DISCUSSION}

This study examined postinjury CVD morbidity in mid to older adults living in NSW, Australia who had an injury serious enough to warrant hospitalisation and found increased rates of hospitalisations for CVD after injury, findings consistent with our previous record linkage research of burn, non-burn trauma and uninjured people. ${ }^{16} 17$ While our previous research included social disadvantage and geographical remoteness indices as proxy measures for lifestyle and health factors, this study used data from the 45 and Up Study which allowed adjustment for individuals' linked medication use, primary care, health and lifestyle factors.

While increasing age was the strongest predictor of postinjury CVD hospitalisations (prevalent and new admissions), those with injury (all types combined) had $19 \%$ (95\% CI 1.14 to 1.25 ) higher rates when compared with the uninjured cohort and spent 40\% (95\% CI 1.13 to 1.66) longer in hospital. These findings are consistent with our previous Western Australian study of postinjury CVD admissions (IRR 1.29, 95\% CI 1.21 to 1.37) and LOS (IRR $1.37,95 \%$ CI 1.13 to 1.66$).{ }^{17}$ Baseline factors associated with significantly reduced postinjury CVD admissions



Figure 4 Unadjusted cardiovascular hospitalisation rates 5 years before and 5 years after injury, by beta blocker (BB) subgroups.

(protective effects) included use of lipid-modifying agents, moderate and vigorous exercise (vs minimal), those within the normal BMI range $\left(18.5-25 \mathrm{~kg} / \mathrm{m}^{2}\right)$ and those who reported a family history of blood pressure. All levels of injury severity (mild, moderate, severe) were associated with significantly elevated postinjury CVD admission rates (mild 20\%, moderate $16 \%$, severe $20 \%$ ), findings also consistent with our previous research. ${ }^{1617}$

Adjusted postinjury admission rates for diagnostic subgroups of CVD indicated higher rates of admissions for IHD $(30 \%)$, HF $(14 \%)$ and cerebrovascular disease (22\%), compared with the uninjured cohort, results that are also in line with previous research. ${ }^{17}$ A $36 \%$ higher rate of first time or incident CVD (total) admissions in the injured cohort was found when compared with the uninjured cohort. In previous research of injury (excluded burns) patients versus uninjured, first time CVD admission rates were highest during the first 6 months (HR $2.19,95 \%$ CI 1.66 to 2.87 ), thereafter for 5 years, the incident rate was 1.16 times higher (95\% CI 1.03 to 1.31$).{ }^{17}$ In this study, higher postinjury incident admission rates were found for IHD (24\%) and cerebrovascular disease (43\%), when compared with the uninjured cohort; however, no significant increase was found for HF. These results differ slightly from previous research that identified higher postinjury (excluded burns) rates for incident IHD,

Table 5 Postinjury cardiovascular disease hospitalisations and record of beta blocker (BB) use yes versus no, among injury cohort: adjusted incidence rate ratios (IRRs) and 95\% Cls

\begin{tabular}{|c|c|c|}
\hline Record of BB medication & $\begin{array}{l}\text { IRR } 95 \% \mathrm{Cl} \text { all cohort } \\
\text { data }\end{array}$ & $\begin{array}{l}\text { IRR } 95 \% \text { Cl subset } \\
\text { cohort data } 2013+\end{array}$ \\
\hline BB 4 months prior and 4 months after injury $n=4329$ (continuous use) & 1.29 (1.17 to 1.42$)$ & 1.31 (1.13 to 1.56$)$ \\
\hline $\begin{array}{l}\text { BB started within } 60 \text { days after injury ( } n=619) \text { (first } 60 \text { days after injury were } \\
\text { excluded from analysis) }\end{array}$ & 1.63 (1.37 to 1.94$)$ & 1.57 (1.22 to 2.03$)$ \\
\hline $\begin{array}{l}\text { BB started within } 120 \text { days after injury ( } n=928) \text { (first } 120 \text { days after injury } \\
\text { were excluded from analysis) }\end{array}$ & 1.63 (1.40 to 1.89$)$ & $1.70(1.35$ to 2.14$)$ \\
\hline
\end{tabular}


cerebrovascular disease and HF; however, rates reached significance only for IHD for 6 months after injury. ${ }^{17}$ However, previous research of incident CVD comparing burn patients with uninjured people, found significantly higher incident rates for IHD, cerebrovascular disease and $\mathrm{HF}$ for at least 5 years after the initial injury. ${ }^{16} 17$

Results from injury subgroup analyses found those with fractures and open wounds had $17 \%$ and $18 \%$ higher postinjury CVD admission rates, respectively. These findings are comparable with our previous results for injury patients in Western Australia for fractures (IRR 1.14, 95\% CI 1.03 to 1.17 ) and open wounds (IRR 1.34, 95\% CI 1.16 to 1.55$)$. However, burn patients had $48 \%$ higher postinjury CVD admission rates (IRR 1.48, 95\% CI 1.02 to 2.15), when compared with the uninjured cohort. This result is consistent with our previous population-based burns research of adults 45 years and older in Western Australia (adjusted IRR 1.46 , 95\% CI 1.36 to 1.56$).{ }^{16}$ Typically, hospitalisation is required for the management of more severe morbidity. The higher adjusted CVD hospitalisation rates found for burn patients may suggest that burns trigger higher levels of systemic endocrine, inflammatory and immune responses with the consequence of greater cardiac stress and/or recovery time of such systemic responses is longer, resulting in greater morbidity and hospitalisation for higher levels of clinical management.

A report of Australian prescription claims in 2014-2015 found the most common cardiovascular system (ATC 'C') medications taken by people with a cardiovascular condition were blood pressure lowering medications (65\%) (including angiotensin II antagonists (19\%), beta blocking agents (18\%), ACE inhibitors (18\%) and lipid-modifying agents $36 \%$ (including statins).$^{28}$ Results presented in table 2 of the distribution of CVD medications used by both injured and uninjured are consistent with these Australian statistics. Examination of prescriptions used in the 4-month period prior to injury (study index date) found renin-angiotensin system and lipidmodifying drugs were the most used in both the injured and uninjured cohorts. However, after injury, when compared with the uninjured cohort, those with injury had significantly increased prescription rates for all ATC groups of cardiovascular medications (combined incident and prevalent use) except antihypertensives (refer to online supplemental table 2) eg, of such medications). Examination of new (incident) prescriptions for CVD in the first 30 days after injury, found the injured cohort had 2.6 times higher use of cardiovascular medications than uninjured adults, with significantly elevated rates for each ATC cardiovascular group except antihypertensives. While the wide $95 \% \mathrm{CI}$ for the subgroups cardiac therapy and calcium channel blockers indicate the small numbers involved, these elevated rates may reflect the acute phase of systemic endocrine, metabolic, inflammatory and immune responses triggered by the injury and subsequent effects on the circulatory system experienced by the injury patient. After 30 days, while with a reduced magnitude, significantly increased incident prescription rates remained for the injury cohort for cardiac therapy (includes cardiac glycosides, antiarrhythmic, adrenergic vasodilators), diuretics and $\mathrm{BB}$ prescriptions when compared with uninjured adults.

Recent evidence suggests that the metabolic and immunomodulatory effects of BBs, ${ }^{512} 29$ a class of medications commonly used to treat CVD, may expand their clinical use in a range of trauma patients. In retrospective studies of patients with trauma, those administered BB (excluded head injuries) during hospital admission (vs no BB) ${ }^{13}$ and within 30 days of intensive care unit admission (vs no BB use), ${ }^{27}$ had significantly lower in-hospital fatality; BB use was identified as an independent protective factor for mortality (adjusted OR, 0.37; $\mathrm{p}=0.007$ ).$^{27} \mathrm{BBs}$ have been found beneficial in paediatric burn patients when used during the acute phase and for up to 1 year to mitigate hypermetabolism and cardiac stress. ${ }^{4}{ }^{140-33}$ In addition, BB administered within 48 hours of burn and continued throughout hospital discharge period (to decrease heart rate by $20 \%$ from admission levels), was found to diminish blood loss during skin grafts surgeries and improved wound healing in adults. ${ }^{26}$

We explored the effects of BB use on postinjury CVD hospital admissions among injury patients who survived their index injury hospitalisation using a triangulation of analytical and graphical methods. The study results which adjusted for an extensive range of health, injury and demographic factors found that continuous use of BB (ie, those using BB continuously pre and post injury vs starting after injury) had reduced postinjury CVD hospitalisation rates. There are several possible interpretations of this result. BBs may have provided beneficial cardioprotective effects with consequent reduced cardiovascular morbidity. BBs typically have a half-life (time it takes for the concentration of the drug in the plasma or the total amount in the body to be reduced by 50\%), of between 3 and 10 hours (hr) (eg, 3-7 hours propranolol, metoprolol; 6-7 hours atenolol; 6-8 hours labetalol; 6-10 hours carvedilol) with clearance of the drug estimated after $4.5 \mathrm{x}$ the respective half-life (hours) ${ }^{34}$ Even in the absence of $\mathrm{BB}$ treatment during the index hospital stay, it is possible that those already on BBs prior to injury had therapeutic plasma levels of BB. For those injury patients on BBs continuously with tight regulation of beta-adrenergic receptors, the BBs may have afforded benefits in managing the acute systemic responses and subsequent physiological effects. A second interpretation is that the difference in cardiovascular morbidity between these groups simply reflects differences in underlying cardiovascular health-individuals already on BBs may have their pre-existing cardiovascular condition under control and so have lower rates of CVD morbidity as compared with those with new-onset BBs, who may have a newly diagnosed condition with greater likelihood of recurrent admissions. Further clinical research of injury patients is required to definitively address this and direct treatment. 


\section{Strengths and limitations}

This study analysed pre-existing data from the 45 and Up Study, a population-based cohort study of 267153 randomly selected adults aged 45 and older in NSW, Australia. The data analysed included self-reported health and lifestyle information linked to hospital (public and private), pharmaceutical and primary care records and indices of geographic remoteness and social disadvantage, providing researchers with the most complete picture of health and illness of these study participants. Our previous record linkage research included all individuals in the population of Western Australia; however, individual-level lifestyle, medication and primary care data were unavailable for linkage including information on many key cardiovascular risk factors, such as smoking, BMI, physical activity, family history, cholesterol and blood pressure. The 45 and Up Study may not be representative of the entire general population of mid to older adults with and without a record of injury admission in NSW; however, the strength of these data is in the ability to support comparison studies within the cohort. While an important strength of this study is the inclusion of linked objective pharmaceutical claims data, it was a study assumption that the supplied medication was consumed. In addition, without linked individual medical records, the precise diagnostic indications for the CVD prescriptions were unknown. Counts and rates of hospitalisations, length of hospital stays, and prescription use are considered valid measures of health outcomes. ${ }^{35}$ While limitations exist with the use of linked health administrative data, such longitudinal data provide a valuable source of information to explore clinical questions at a population level with a comparison cohort. ${ }^{36}$

While our controls were matched on sex and age, imbalances remained between the cohorts, particularly in regard to previous health status. These include difference in the patterns of health service use; higher service use may be indicative of poor health, or higher healthseeking behaviour, while lower service use may be indicative of better health or healthcare avoidance. Those with injury hospitalisation have higher rates of cardiovascular admissions compared with the controls prior to have an injury, suggesting the potential for bias. In addition, there was no evidence of a dose/response relationship, with those with more severe injuries not having any greater cardiovascular morbidity when compared with those with more minor injuries. To ensure the validity of our conclusions, a propensity score analysis was undertaken to further investigate our main model which showed higher cardiovascular hospitalisations in those with injury diagnosis. Propensity score analysis allowed us to account for imbalances in confounders between cohorts, although only a minimal number of covariates can be included. To address these potential imbalances, we included confounders pertaining to prior health status, and health service utilisation. Results from this analysis replicated our main finding, providing additional strength to our conclusions. However, controlling for all confounders is difficult in an observational design with imbalanced groups, and as such the data provides only weak evidence to support our conclusions. A randomised design is required to gain further confidence.

Our investigation of BBs effect on cardiovascular hospitalisations was exploratory, with our findings suggestive of multiple competing hypotheses that could not be disentangled. Our study investigated the effect of BBs on cardiovascular hospitalisations but provided no detailed analysis of other cardiovascular medications. While there are reasons to expect BBs to have greater cardioprotective effects than other cardiovascular medications, ${ }^{529}$ these other medications also have anti-inflammatory effects and may contribute to a purported cardio-protective effect. A large portion of our study sample were taking multiple cardiovascular medications, making the effect of one single medication difficult to isolate.

\section{CONCLUSION}

This study analysed self-reported health and lifestyle data of participants in an Australian population-based study linked with hospital admissions, pharmaceutical claims and primary care data to investigate the effect of injury on longer term postinjury CVD hospitalisations. Findings of increased postinjury hospitalisation rates and prolonged length of hospital stay related to CVD in the injury cohort are consistent with previous research and may suggest that injury has long-lasting systemic impacts on the heart and circulation, requiring prolonged clinical support. While our analyses of BB use among injury patients appeared to show reduced need for CVD hospitalisation by those injury patients who were on continuous BB treatment (preinjury and postinjury), further definitive clinical research is required to determine causality.

Acknowledgements This research was completed using data collected through the 45 and Up Study (www.saxinstitute.org.au). The 45 and Up Study is managed by the Sax Institute in collaboration with major partner Cancer Council NSW; and partners: the National Heart Foundation of Australia (NSW Division); NSW Ministry of Health; NSW Government Family \& Community Services-Ageing, Carers and the Disability Council NSW; and the Australian Red Cross Blood Service. We thank the many thousands of people participating in the 45 and Up Study. The authors thank the data custodians of the core health datasets of the Department of Health NSW for access to data and the staff of the Centre for Health Record Linkage NSW for data extraction and linkage, the staff of the 45 and Up Study for access and linkage of their project data, and the Department of Human Services for access to MBS and PBS data. The authors thank Amira Allaham, PhD candidate Burn Injury Research Unit UWA, for designing figure1.

Contributors All authors have made contributions to the paper and authorised the submission: JMD designed the study, provided data interpretation and drafted manuscript. SMR conducted analyses and drafted manuscript. JB assisted in study design. MWF, SR and FMW contributed clinical interpretation. All authors contributed to manuscript preparation and critical revision.

Funding This work was supported by The Fiona Wood Foundation. Grant number: not applicable.

Competing interests None declared.

Patient consent for publication Not required.

Ethics approval This retrospective cohort project was approved by the New South Wales (NSW) Population and Health Services Research Ethics Committee and Curtin 
University Human Research Ethics Committee. The 45 and Up Study was approved by the University of New South Wales Human Research Ethics Committee.

Provenance and peer review Not commissioned; externally peer reviewed.

Data availability statement № additional data are available.

Supplemental material This content has been supplied by the author(s). It has not been vetted by BMJ Publishing Group Limited (BMJ) and may not have been peer-reviewed. Any opinions or recommendations discussed are solely those of the author(s) and are not endorsed by BMJ. BMJ disclaims all liability and responsibility arising from any reliance placed on the content. Where the content includes any translated material, BMJ does not warrant the accuracy and reliability of the translations (including but not limited to local regulations, clinical guidelines, terminology, drug names and drug dosages), and is not responsible for any error and/or omissions arising from translation and adaptation or otherwise.

Open access This is an open access article distributed in accordance with the Creative Commons Attribution Non Commercial (CC BY-NC 4.0) license, which permits others to distribute, remix, adapt, build upon this work non-commercially, and license their derivative works on different terms, provided the original work is properly cited, appropriate credit is given, any changes made indicated, and the use is non-commercial. See: http://creativecommons.org/licenses/by-nc/4.0/.

ORCID iD

Sean M Randall http://orcid.org/0000-0002-2756-5090

\section{REFERENCES}

1 Foex BA. Systemic responses to trauma. Br Med Bull 1999;55:726-43.

2 Johnson BZ, McAllister S, McGuire HM, et al. Pediatric burn survivors have long-term immune dysfunction with diminished vaccine response. Front Immunol 2020;11:1-15.

3 O'Halloran E, Shah A, Dembo L, et al. The impact of non-severe burn injury on cardiac function and long-term cardiovascular pathology. Sci Rep 2016;6:34650.

4 Finnerty CC, Herndon DN. Is propranolol of benefit in pediatric burn patients? Adv Surg 2013;47:177-97.

5 Loftus TJ, Efron PA, Moldawer LL, et al. Beta-blockade use for traumatic injuries and immunomodulation: a review of proposed mechanisms and clinical evidence. Shock 2016;46:341-51.

6 Molina PE. Neurobiology of the stress response: contribution of the sympathetic nervous system to the neuroimmune axis in traumatic injury. Shock 2005;24:3-10.

7 Williams FN, Herndon DN, Suman OE, et al. Changes in cardiac physiology after severe burn injury. J Burn Care \& Res 2011;32:269-74.

8 Friese RS, Barber R, McBride D, et al. Could beta blockade improve outcome after injury by modulating inflammatory profiles? J Trauma 2008;64:1061-8.

9 Jetschmann J-U, Benschop RJ, Jacobs R, et al. Expression and in-vivo modulation of $\alpha$ - and $\beta$-adrenoceptors on human natural killer (CD16+) cells. J Neuroimmunol 1997;74:159-64.

10 Oberbeck R, Kobbe P. Beta-Adrenergic antagonists: indications and potential immunomodulatory side effects in the critically ill. Current Med Chemist 2009;16:1082-90.

11 Auerbach AD, Goldman L. Beta-blockers and reduction of cardiac events in noncardiac surgery:clinical applications. JAMA 2002;287:1445-7.

12 Elenkov IJ, Wilder RL, Chrousos GP, et al. The sympathetic nerve-an integrative interface between two supersystems: the brain and the immune system. Pharmacol Rev 2000;52:595-638.
13 Arbabi S, Campion EM, Hemmila MR, et al. Beta-blocker use is associated with improved outcomes in adult trauma patients. $J$ Trauma 2007;62:56-62

14 Herndon DN, Hart DW, Wolf SE, et al. Reversal of catabolism by beta-blockade after severe burns. N Eng J Med 2001;345:1223-9.

15 Duke JM, Randall SM, Fear MW, et al. Long-Term effects of pediatric burns on the circulatory system. Pediatrics 2015;136:e1323-30.

16 Duke JM, Randall SM, Fear MW, et al. Understanding the longterm impacts of burn on the cardiovascular system. Burns 2016;42:366-74.

17 Duke JM, Randall SM, Fear MW, et al. Long term cardiovascular impacts after burn and non-burn trauma: a comparative populationbased study. Burns 2017;43:1662-72.

18 Banks E, Redman S, Jorm L, et al. Cohort profile: the 45 and up study. Int J Epidemiol 2008;37:941-7.

19 Stephenson SCR, Langley JD, Civil ID. Comparing measures of injury severity for use with large databases. J Trauma 2002;53:326-32.

20 WHO Collaborating Centre for Drug Statistics Methodology. Guidelines for ATC classificationand DDD assignment 2013. Oslo: WHO, 2012.

21 Charlson ME, Pompei P, Ales KL, et al. A new method of classifying prognostic comorbidity in longitudinal studies: development and validation. J Chronic Dis 1987;40:373-83.

22 Trewin D. Socio-economic indexes for areas Australia. (information paper, census of population and housing). cat. No. 2039.0. Canberra: Australian Bureau of Statistics, 2003

23 Glover J, Tennant S. Remote areas statistical geography in Australia: notes on the accessibility/remoteness index for Australia (ARIA+ version). Working papers series No. 9 Adelaide: public health information development unit, Adelaide, the University of Adelaide, 2003.

24 Posner MA, Ash AS. Comparing weighting methods in propensity score analysis 2012. Available: http://www.stat.columbia.edu/ gelman/stuff for blog/posner.pdf [Accessed 22 Sept 2020].

25 Becker SO, Ichino A. Estimation of average treatment effects based on propensity scores. Stata J 2002;2:358-77.

26 Ali A, Herndon DN, Mamachen A, et al. Propranolol attenuates hemorrhage and accelerates wound healing in severely burned adults. Crit Care 2015;19:217.

27 Bukur M, Lustenberger T, Cotton B, et al. Beta-Blocker exposure in the absence of significant head injuries is associated with reduced mortality in critically ill patients. Am J Surg 2012;204:697-703.

28 Australian Institute of Health and Welfare. Medicines for cardiovascular disease. cat. No. CVD 80. Canberra: AlHW, 2017.

29 Flores O, Stockton K, Roberts JA, et al. The efficacy and safety of adrenergic blockade after burn injury: a systematic review and metaanalysis. J Trauma Acute Care Surg 2016;80:146-55.

30 Baron PW, Barrow RE, Pierre EJ, et al. Prolonged use of propranolol safely decreases cardiac work in burned children. J Burn Care Rehab 1997;18:223-7.

31 Herndon DN, Rodriguez NA, Diaz EC, et al. Long-term propranolol use in severely burned pediatric patients: a randomized controlled study. Annals of Surgery 2012;256:402-11.

32 Porter C, Tompkins RG, Finnerty CC, et al. The metabolic stress response to burn trauma: current understanding and therapies. Lancet 2016;388:1417-26.

33 Williams FN, Herndon DN, Kulp GA, et al. Propranolol decreases cardiac work in a dose-dependent manner in severely burned children. Surgery 2011;149:231-9.

34 Koda-Kimble MA, Alldredge BK, Corelli RL, et al. Applied Therapeutics. In: The clinical use of drugs. 9th edn. Philadelphia: Lippincott, williams \& Wilkins, 2009.

35 Hendrie D, Miller TR. Assessing the burden of injuries: competing measures. Inj Control Saf Promot 2004;11:193-9.

36 Cohen MM, MacWilliam L. Measuring the health of the population. Med Care 2003;33:DS21-42. 